\title{
Associations between nasopharyngeal carriage of Group B Streptococcus and other respiratory pathogens during early infancy
}

Ebenezer Foster-Nyarko ${ }^{1}$, Brenda Kwambana ${ }^{1}$ Odutola Aderonke ${ }^{1}$, Fatima Ceesay ${ }^{1}$, Sheikh Jarju', Abdoulie Bojang ${ }^{1}$, Jessica McLellan ${ }^{1}$, James Jafali ${ }^{1}$, Beate Kampmann ${ }^{1}$, Martin O. Ota ${ }^{1,2}$, Ifedayo Adetifa ${ }^{3,4}$ and Martin Antonio 1,5,6*

\begin{abstract}
Background: In West Africa, the carriage of Group B Streptococcus (GBS), among infants is poorly characterised. We investigated co-carriage of GBS with other respiratory pathogens in the infants' nasopharynx in The Gambia.

Methods: We assessed the carriage, serotypes and antibiotic susceptibility of Beta-haemolytic Streptococci (BHS) groups A-G; along with the carriage of Streptococcus pneumoniae; Haemophilus influenzae; Staphylococcus aureus and Moraxella catarrhalis in 1200 two-month old infants.

Results: The BHS prevalence was $20.0 \%$ and GBS dominated (13.8\%), particularly serotypes $V$ and II; serotype V being negatively associated with $\mathrm{H}$. Influenzae carriage (OR 0.41 [95 \% Cl: 0.18-0.93], $p=0.033$ ). Although co-colonization of GBS and other BHS was not seen, colonization with GBS was positively associated with S. aureus (OR 1.89 [95 \% Cl: 1. 33-2.69], $P<0.001$ ) and negatively associated with S. pneumoniae (OR 0.47 [95 \% Cl: 0.33-0.67], $\mathrm{p}<0.001$ ) and $\mathrm{M}$. catarrhalis (OR 0.61 [95 \% Cl: 0.40-0.92], $p=0.017$ ). $\geq 89 \%$ of GBS isolates were susceptible to most antibiotics tested, except for tetracycline resistance, which was $89 \%$.

Conclusion: This study provides baseline data on the carriage of GBS in two month old infants from West Africa. The dominant serotypes of GBS in this setting are serotypes $V$ and II. This may be important for future GBS vaccine development for the West African sub-region.
\end{abstract}

Keywords: Nasopharyngeal, Group B streptococcus, Beta-haemolytic streptococci, Gambia

\section{Background}

The beta-haemolytic group of Streptococci (BHS) causes a wide variety of infections ranging from mild skin infections to life threatening diseases including sepsis and meningitis $[1,2]$. BHS are classified into Lancefield serogroups (A -G) depending on their cell wall structure [3]. BHS are amongst the top ten causes of invasive bacterial diseases in adults and children beyond the neonatal period $[4,5]$.

\footnotetext{
* Correspondence: mantonio@mrc.gm

${ }^{1}$ Vaccines and Immunity Theme, Medical Research Council Unit, Banjul, The Gambia

${ }^{5}$ Faculty of Infectious and Tropical Diseases, London School of Hygiene \&

Tropical Medicine, London, UK

Full list of author information is available at the end of the article
}

A sub-group of the BHS is Group B Streptococcus (GBS) also known as Streptococcus agalactiae, which is a major cause of neonatal sepsis and meningitis with significant morbidity and mortality rates in the developed world [6, 7]. However, there are historical and now emerging data suggesting that GBS may also be a frequent cause of neonatal sepsis in sub-Saharan Africa [810]. GBS comprises ten serotypes (Ia, Ib, II-IX) and there are geographical variations with respect to the predominant serotypes in invasive disease $[7,11,12]$.

Microbes colonizing the nasopharynx can invade the blood stream or migrate to contiguous surfaces of the respiratory tract [13]. Consequently, nasopharyngeal carriage of respiratory pathogens such as $S$. pneumoniae is 
a risk factor for the development of several infections including pneumonia, meningitis and sepsis [14]. Droplet secretions from the nasopharynx are also an important mechanism for the horizontal transfer of pathogens [15]. The epidemiology, transmission and nasopharyngeal carriage of S. pneumoniae and Haemophilus influenzae type b (Hib) have been studied in the Gambia [16, 17]. However, nasopharyngeal co-carriage of GBS with other respiratory pathogens in the post pneumococcal and $\mathrm{Hib}$ conjugate vaccine era has not been described in West Africa.

This study investigated co-carriage of GBS with other BHS and four common respiratory pathogens colonizing the nasopharyngeal mucosae beyond the first week of life. We also characterised the carriage, serotypes and antibiotic susceptibility of GBS in these Gambian infants who had not yet been vaccinated with the pneumococcal conjugate and Hib vaccines. This study provides critical baseline data on the carriage of GBS and other BHS in the sub-region.

\section{Methods}

\section{Study area and population}

The study was conducted in the Fajikunda district, a typical peri-urban setting in the Western region of The Gambia. As described previously, this area is representative of similar communities situated near large cities in West Africa [18].

Nasopharyngeal swabs (NPS) were obtained between July 2011 and May 2012 from 1200 healthy infants aged two months of consenting parents at the Fajikunda infants' well-being clinic.

The Gambia Government/MRC Joint Ethics Committee approved the study. Trained field workers/nurses explained the contents of the study information sheet to parents/guardians in their own language. Parents/guardians of all study participants gave written informed consent prior to enrolment.

The study team administered questionnaires to record history of acute respiratory tract infection, place of birth (whether in a health facility or at home), cooking method, ethnic group, number of other infants less than 5 years in the household and sex.

\section{Nasopharyngeal swab sampling methods}

NPS were collected as described previously [18] and were processed for the isolation of BHS and four additional respiratory pathogens including S. pneumoniae, $H$. influenzae, S. aureus and M. catarrhalis.

\section{Microbiological methods}

Identification and isolation of bacterial pathogens

The broth enrichment method for enhancing the yield of S. pneumoniae [19] was adapted to improve the yield of BHS and other respiratory pathogens. Briefly, NPS stored in Skim-milk Tryptone Glucose Glycerol were thawed at room temperature $\left(25^{\circ} \mathrm{C}\right)$ and briefly vortexed. $200 \mu \mathrm{l}$ of the NPS in Skim-milk Tryptone Glucose Glycerol was inoculated into 5mls of Todd-Hewitt Broth with $5 \%$ Yeast Extract (Oxoid, Basingstoke, UK) containing $1 \mathrm{ml}$ of rabbit serum (B\&K Universal Ltd, Grimston, East Yorkshire, UK) and incubated for $5-6 \mathrm{~h}$ at $37{ }^{\circ} \mathrm{C}$ in ambient air.

For BHS isolation, $100 \mu \mathrm{l}$ aliquots were streaked onto a $5 \%$ Sheep Blood Agar plate containing a 1:50000 dilution of crystal violet to inhibit Staphylococcus growth. For M. catarrhalis, H. influenzae, S. pneumoniae and S. aureus isolation, aliquots of $100 \mu \mathrm{l}$ were streaked onto each of Chocolate agar, Bacitracin chocolate agar, Gentamicin sheep blood agar and Mannitol salt agar plates, respectively. All plates were incubated overnight at $37{ }^{\circ} \mathrm{C}$; Gentamicin sheep blood agar, Bacitracin chocolate agar and Chocolate agar plates in $5 \% \mathrm{CO}_{2}$; Crystal violet blood agar and Mannitol Salt agar in ambient air.

Isolation and identification of bacterial pathogens $(H$. influenzae, S. pneumoniae, M. catarrhalis and S. aureus) were carried out as previously reported [18]. For $H$. influenzae; type B specific antisera obtained from Statens Serum Institut (SSI, Copenhagen, Denmark) was used to serotype the isolates as Hib or non-Hib.

Isolation of BHS was carried out as follows. Following overnight incubation of the Crystal violet blood agar plates, 2-3 colourless or grey, dry or moist, betahaemolytic colonies were individually streaked to Blood agar plates. A 0.04 Units bacitracin disk (BD Oxoid, Basingstoke, UK) was placed in the first quadrant for the preliminary identification of Group A Streptococcus (GAS). The plates were then incubated overnight at $37^{\circ}$ $\mathrm{C}$ in $5 \% \mathrm{CO}_{2}$. All bacitracin negative, catalase negative colonies were subjected to the Christie, Atkins, Munch and Peterson test for the preliminary identification of GBS. S. agalactiae (ATCC 12386) were inoculated as negative and positive controls, respectively, for each batch of testing. All beta-haemolytic isolates (including all isolates preliminarily identified as GAS and GBS) were further grouped using the Streptex ${ }^{\circ}$ Streptococcal grouping kit (Remel \& Oxoid, Thermo Fisher Scientific, Basingstoke, UK) for confirmation as GAS, GBS, Group C (GCS) or group G (GGS). All isolates were stored in $15 \%$ Glycerol broth at $-70{ }^{\circ} \mathrm{C}$ until further testing.

\section{Serotyping of GBS isolates \\ Latex agglutination method}

One hundred thirty-three out of 162 (82\%) GBS isolates were successfully revived for serotyping by the slide latex agglutination method. Confirmed GBS isolates were streaked onto a $5 \%$ Blood agar plate to obtain fresh cultures. Serotyping was carried out using Strep-B-latex 
antisera $^{\circ}$ (SSI, Copenhagen, Denmark) as per manufacturer's instructions. For each batch of serotyping, serotype specific GBS controls strains Ia, Ib, II - IX obtained from Centres for Disease Control and Prevention, Atlanta (Strain numbers 2008232728, 2008232729, 2008232738, 2008232582, 2011201884, 2008232731, 2010228816, 4832-06, 5030-08, 7509-07 respectively) [20] were used as positive controls.

\section{Conventional PCR serotyping method}

All the GBS strains which were non-typeable (NT) by the latex agglutination method (21/133, $15.8 \%$ ) were subjected to the conventional serotyping method (Polymerase Chain Reaction, PCR). In addition, $15.0 \%$ of all the GBS strains typed by the latex agglutination method (20/133) were confirmed by PCR.

DNA was extracted from pure overnight colonies of GBS following as per the World Health Organization (WHO) Laboratory manual for the diagnosis of meningitis [21].

Serotyping was then carried out using the method of Imperi et al. [20]. For each run, non-template negative controls as well as positive GBS control strains (serotypes Ia, Ib, II-IX (Centres for Disease Control and Prevention, Atlanta, USA) were included.

\section{Susceptibility testing}

All the 133 GBS isolates that were serotyped were further tested for their susceptibility to Penicillin (10 units), Chloramphenicol $(30 \mu \mathrm{g})$, Tetracycline $(30 \mu \mathrm{g})$ and Erythromycin $(15 \mu \mathrm{g})$ discs (BD Oxoid, Basingstoke, United Kingdom) by the Kirby-Bauer method. E-tests (Biomerieux, Basingstoke, United Kingdom Limited) were performed for all isolates showing resistant or intermediate sensitivities by the Kirby-Bauer method.

All interpretation of antibiotic susceptibilities were done using CLSI breakpoints [22].

All reagents and the disc dispenser were obtained from BD Oxoid, Basingstoke, UK, except otherwise stated.

The MRC Unit The Gambia, molecular microbiology laboratory submits to the external quality assurance programme of the UK National External Quality Assessment Service [23] and is a World Health Organization (WHO) Regional Reference Laboratory for invasive bacterial pathogens.

\section{Statistical analysis}

Data management was done using SQL integrated database with a Microsoft Access front-end linked to field, clinical and laboratory databases.

All statistical analyses were conducted in Stata version 12 for Windows (Stata Corp, College Station, TX). Any association with a $\mathrm{p}$ value of $<0.05$ was considered to be significant.
Categorical variables were summarised using frequency counts and percentages, $\mathrm{n}(\%)$. Mean (SD) and median (IQR) values were used to describe normally and nonnormally distributed continuous variables, respectively.

Cross tabulations (with Fishers' exact tests for associations) were used to describe and compare the prevalence of BHS carriage across different levels of individual potential risk factors including carriage of other pathogens (categorical variables). Distributions of continuous variables were compared between BHS carriers and noncarriers using independent sample $\mathrm{t}$-test (if approximately normal) or non-parametric Wilcoxon rank-sum test (if non-normal).

Univariable (simple) and multivariable (multiple) logistic regression analyses were then performed to estimate unadjusted and adjusted odds ratios (respectively) with their $95 \%$ confidence intervals to quantify the associations of BHS carriage prevalence with the potential risk factors. The confounding effects of other variables and presence of statistical interactions were also assessed during multiple logistic regression analyses. The combination of risk factors mutually associated with BHS was identified using a stepwise backward elimination (logistic regression) procedure, with an exclusion $p$ value of 0.1 .

\section{Results}

There were 1200 participants aged 2 months of which 1170 (97.5 \%) had complete data for analysis. The algorithm for recruitment and sample processing flow is shown in Fig. 1. The characteristics of the study population are summarized in Table 1.

\section{Prevalence of bacterial species}

Figure 2a shows carriage of BHS and 4 common respiratory pathogens in the nasopharynx; with $S$. pneumoniae being the highest $(729 / 1170,62.3 \%)$ and $H$. influenzae being the least $(368 / 1170,31.5 \%)$ in carriage respectively. All the $H$. influenzae isolates recovered were serotyped to be non-Hib.

Of 234 out of $1170(20.0 \%)$ BHS isolated, GBS was the most dominant $(162 / 234,69.2 \%)$, and GCS the least prevalent (12/234; $5.1 \%$ ) (Fig. 2b). Two isolates that could not be assigned to any Lancefield group (BHS non-typeable) were excluded from the overall prevalence and risk factor analyses. Similar proportions of males and females carried BHS in their nasopharynx (127/579 (21.9\%) and 107/591 (18.1\%) respectively).

\section{Serotype distribution of GBS}

Of the 162 GBS isolates that were recovered, 133 ( $\approx 2 \%$ ) were successfully revived for serotyping.

Nine different GBS serotypes were identified of which the most prevalent were serotypes V (50/133, $37.6 \%)$ 


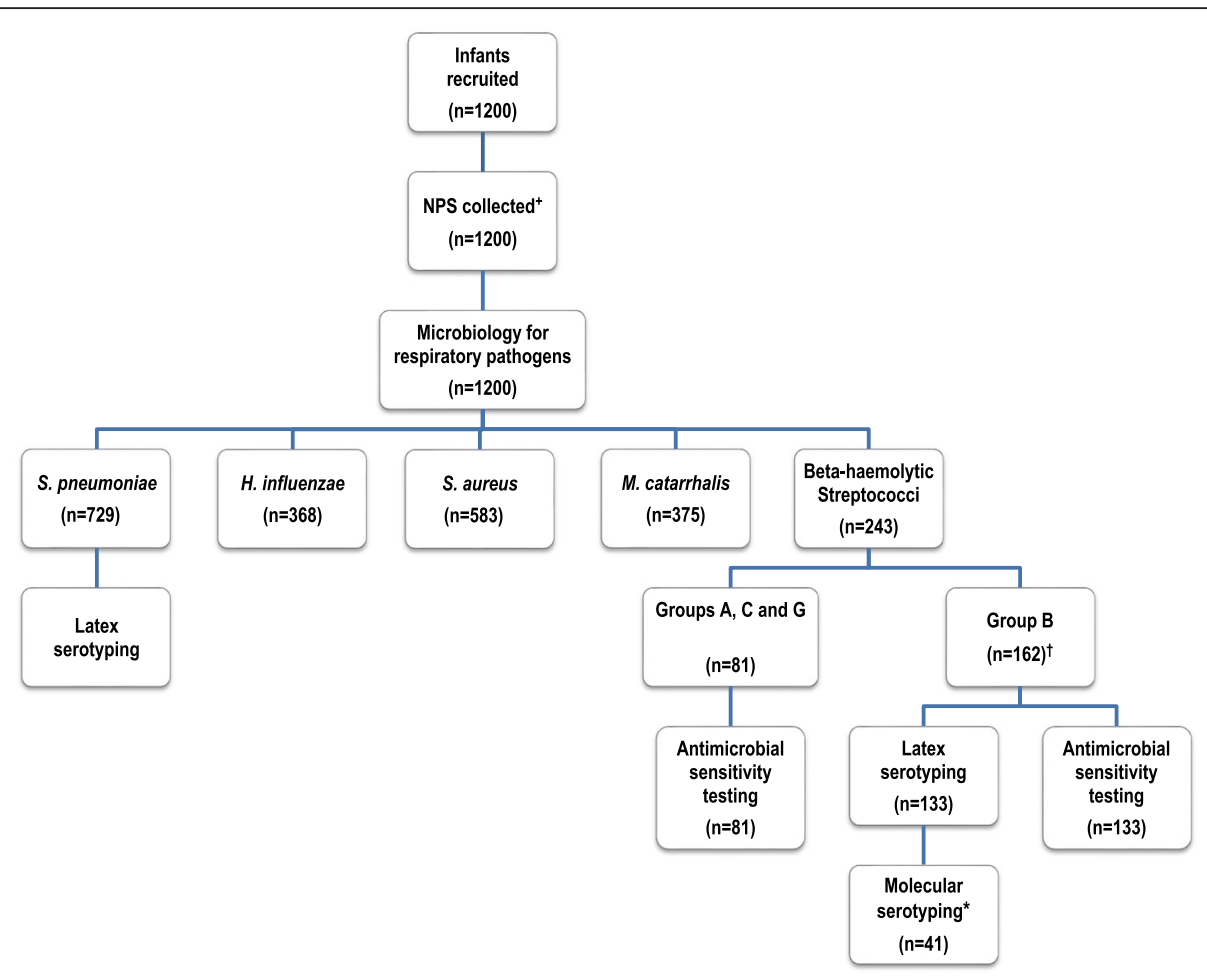

Fig. 1 Algorithm for recruitment and sample processing flow. ${ }^{+} 1200$ infants aged 2 months of consenting mothers were recruited for the study; however 1170 (97.5\%) had complete data for analysis. ${ }^{\dagger} 162 \mathrm{GBS}$ isolates were recovered in the study, however 133 (82 \%) were successfully revived for serotyping. ${ }^{*} 21$ GBS isolates were non-typeable (NT) by the latex agglutination method and were subjected to the conventional Polymerase Chain Reaction serotyping method. In addition, 20 isolates typed by the latex agglutination method (15\%) were confirmed by PCR

Table 1 Demographic characteristic of 1170 Gambian 2-month old infants participants

\begin{tabular}{|c|c|c|}
\hline Characteristic & n (\%) & $\%$ \\
\hline \multicolumn{3}{|l|}{ Sex } \\
\hline Male & 579 & 49.5 \\
\hline Female & 591 & 50.5 \\
\hline \multicolumn{3}{|l|}{ Place of birth } \\
\hline Other ${ }^{\mathrm{b}}$ & 98 & 8.4 \\
\hline Health Facility & 1072 & 91.6 \\
\hline \multicolumn{3}{|l|}{ Sleep Rmª } \\
\hline 0 & 374 & 32.4 \\
\hline 1 & 433 & 37.5 \\
\hline 2 & 214 & 18.5 \\
\hline $3^{\mathrm{b}}$ & 134 & 11.6 \\
\hline \multicolumn{3}{|c|}{ History of previous antibiotic use } \\
\hline No & 1170 & 100 \\
\hline Yes & 0 & 0 \\
\hline \multicolumn{3}{|l|}{ History of ARI } \\
\hline No & 1041 & 89.0 \\
\hline Yes & 129 & 11.0 \\
\hline
\end{tabular}

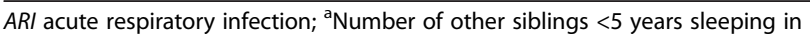
the same room with subject (data available for 1155 infants); ${ }^{\text {bOther, At home/ }}$ delivered by a traditional birth attendant and II (46/133, $34.6 \%)$, which together constituted approximately $72 \%$ of all the GBS serotypes identified. The other GBS serotypes were Ia (10/133, $7.5 \%)$, IV (8/ 133, $6.0 \%)$, Ib (7/133, $5.3 \%)$, VII (5/133, $3.8 \%)$, VIII (1/ 133, $0.8 \%)$, III (1/133, $0.8 \%)$ and VI (1/133, $0.8 \%)$ (Table 2). Serotyping by PCR resolved all strains that were non-typeable by latex agglutination, except for 2 isolates $(2 / 133,1.5 \%)$. Two infants were colonized with two different serotypes (V \& VII, and V \& VIII respectively).

The PCR method was in agreement with the latex agglutination method in the $15.0 \%$ (20 isolates) that were confirmed by PCR.

\section{Risk factors for BHS carriage}

In univariate and multivariable logistic regression analyses of risk factors for BHS carriage, carriage of the Streptococcus groups A-G was not associated with gender, ethnicity, home or hospital delivery, or number of other siblings $<5$ years sleeping in the same room as the participating infant. There were no significant associations between the carriage of GAS or GCS and a history of acute respiratory infection. In contrast, a history of acute respiratory infection was 


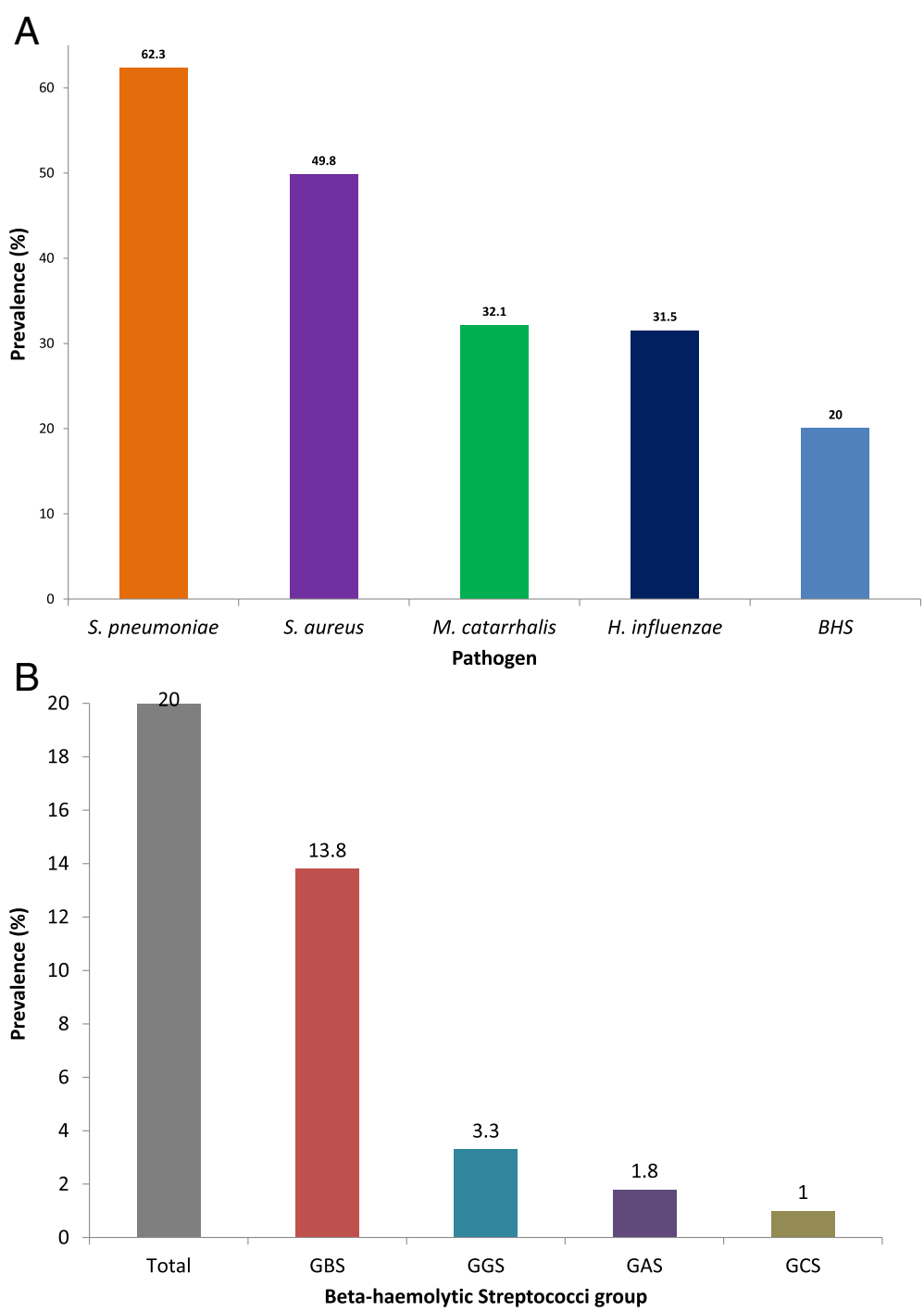

Fig. 2 a Nasopharyngeal carriage of respiratory pathogens in 2-month old Gambian infants. b Nasopharyngeal carriage of Beta-haemolytic Streptococci In 2-month old Gambian infants. BHS - Beta-haemolytic Streptococci; GBS - Group B Streptococci; GGS - Group G; Streptococci; GAS - Group A Streptococci; GCS - Group C Streptococci

significantly associated with carriage of GGS (OR 2.42, [95 \% CI: 1.12-5.23], $p=0.025$ ).

As shown in Table 3, GBS carriage was negatively associated with a history of acute respiratory infection (OR 0.28, [95 \% CI: 0.13-0.63], $p=0.002$ ).

\section{Co-carriage of GBS and other pathogens}

GBS was not carried along with any other BHS in our study population. GBS co-colonization was most frequent with $S$. aureus (104/583, $17.8 \%$ ), followed by H. influenzae (38/368, $10.3 \%)$, then S. pneumoniae

Table 2 Temporal changes in the Group B Streptococcus Serotype distribution from the same geographic region (Fajikunda) in The Gambia: 20 years apart

\begin{tabular}{|c|c|c|c|c|c|c|c|c|c|c|c|c|}
\hline \multirow[t]{2}{*}{ Study } & \multirow[t]{2}{*}{ Year } & \multirow[t]{2}{*}{ Description } & \multirow{2}{*}{$\begin{array}{l}\text { Total number } \\
\text { of isolates }\end{array}$} & \multicolumn{9}{|c|}{$\%$ with serotype } \\
\hline & & & & la & $\|$ & III & IV & V & $\mathrm{Vl}$ & VII & VIII & $\overline{\mathrm{NT}}$ \\
\hline Ref 24 & May 1992 to February 1993 & Maternal and infant colonization & $32^{a}$ & 19 & 28 & 6 & 3 & 38 & - & $-b$ & $-b$ & 6 \\
\hline Present study & July 2011 and May 2012 & Infant colonization & 133 & 7.5 & 34.6 & 0.8 & 6.0 & 37.6 & 0.8 & 3.8 & 0.8 & 1.5 \\
\hline
\end{tabular}

atancefield grouping was performed for only serotypes I-VI and did not include serotypes VI-IX 
Table 3 Risk factors for carriage of Group B Streptococcus

\begin{tabular}{|c|c|c|c|c|c|c|c|}
\hline \multirow[t]{2}{*}{ Risk factor } & \multirow[t]{2}{*}{ Category } & \multirow[t]{2}{*}{$\mathrm{N}$} & \multirow{2}{*}{$\begin{array}{l}\text { Positivity of outcome } \\
\mathrm{n}(\%)\end{array}$} & \multicolumn{2}{|c|}{ Univariable analysis } & \multicolumn{2}{|c|}{ Multivariable analysis } \\
\hline & & & & OR $(95 \% \mathrm{Cl})$ & $p$ value & OR (95 \% Cl) & $p$ value \\
\hline \multirow[t]{2}{*}{ Sex } & Male & 579 & $90(15.54)$ & 1 & & 1 & \\
\hline & Female & 591 & $72(12.18)$ & $0.75(0.54 ; 1.05)$ & 0.097 & $0.73(0.52 ; 1.04)$ & 0.08 \\
\hline \multirow[t]{2}{*}{ Place of Birth } & Other & 98 & $9(9.18)$ & 1 & & & \\
\hline & $H / F$ & 1072 & $153(14.27)$ & $1.65(0.81 ; 3.34)$ & 0.167 & - & - \\
\hline \multirow[t]{5}{*}{ Tribe } & Mandika & 463 & $62(13.39)$ & 1 & & & \\
\hline & Wolof & 164 & $22(13.41)$ & $1(0.59 ; 1.69)$ & 0.994 & - & - \\
\hline & Fula & 144 & 19 (13.19) & $0.98(0.57 ; 1.71)$ & 0.952 & - & - \\
\hline & Jola & 215 & $24(11.16)$ & $0.81(0.49 ; 1.34)$ & 0.418 & - & - \\
\hline & Others & 184 & $35(19.02)$ & $1.52(0.96 ; 2.39)$ & 0.072 & - & - \\
\hline \multirow[t]{4}{*}{ *sleep Room } & 0 & 374 & $59(15.78)$ & 1 & & & \\
\hline & 1 & 433 & $58(13.39)$ & $0.83(0.56 ; 1.22)$ & 0.339 & - & - \\
\hline & 2 & 214 & $28(13.08)$ & $0.8(0.49 ; 1.31)$ & 0.377 & - & - \\
\hline & $3+$ & 134 & $14(10.45)$ & $0.62(0.34 ; 1.16)$ & 0.134 & - & - \\
\hline \multirow[t]{2}{*}{ History of ARI } & 0 & 1041 & 155 (14.89) & 1 & & 1 & \\
\hline & 1 & 129 & $7(5.43)$ & $0.33(0.15 ; 0.72)$ & 0.005 & $0.28(0.13 ; 0.63)$ & 0.002 \\
\hline \multirow[t]{2}{*}{ S. pneumoniae } & Negative & 441 & 88 (19.95) & 1 & & 1 & \\
\hline & Positive & 729 & 74 (10.15) & $0.45(0.32 ; 0.63)$ & $<0.001$ & $0.47(0.33 ; 0.67)$ & $<0.001$ \\
\hline \multirow[t]{2}{*}{ H. influenzae } & Negative & 802 & $124(15.46)$ & 1 & & & \\
\hline & Positive & 368 & 38 (10.33) & $0.63(0.43 ; 0.93)$ & 0.019 & - & - \\
\hline \multirow[t]{2}{*}{ S. aureus } & Negative & 587 & $58(9.88)$ & 1 & & 1 & \\
\hline & Positive & 583 & $104(17.84)$ & $1.98(1.4 ; 2.79)$ & $<0.001$ & $1.89(1.33 ; 2.69)$ & $<0.001$ \\
\hline \multirow[t]{2}{*}{ M. catarrhalis } & Negative & 795 & $126(15.85)$ & 1 & & 1 & \\
\hline & Positive & 375 & $36(9.6)$ & $0.56(0.38 ; 0.84)$ & 0.004 & $0.61(0.40 ; 0.92)$ & 0.017 \\
\hline
\end{tabular}

$H / F$ health facility, ARI acute respiratory infection; *Number of other siblings $<5$ years sleeping in the same room with subject. (-) indicates variables which were not included in the multivariate analysis

(74/729, $10.2 \%)$, and M. catarrhalis (36/375, $9.6 \%)$ (Table 3).

\section{Association between BHS and four common respiratory pathogens}

As shown in Table 3, carriage of GBS was negatively associated with that of S. pneumoniae and M. catarrhalis but positively associated with carriage of S. aureus (OR 1.89 [95 \% CI: 1.33-2.69], $p<0.001)$. Although carriage of GBS was also negatively associated with that of $H$. influenzae in the univariate analysis this association was not confirmed in multivariable analysis. We also found a significant negative association between GBS serotype $\mathrm{V}$ and the carriage of $H$. influenzae (OR 0.41 [95 \% CI: $0.18-0.93], p=0.033)$.

\section{Antibiotic susceptibility of GBS}

All the GBS isolates tested were susceptible to Penicillin and $97.0 \%(129 / 133)$ were susceptible to Chloramphenicol. Resistance was highest to Tetracycline (119/133,
$89.5 \%)$ with a minority of isolates resistant to Erythromycin (1/133, $0.8 \%)$.

\section{Discussion}

To our knowledge, this is the first report of co-carriage of GBS with other respiratory pathogens in the nasopharynx of two-month old infants. These data also provide an update on the circulating serotypes of GBS, as available data from West Africa are from the 1990's [24]. Additionally, we describe for the first time, the nasopharyngeal prevalence of other GBS and BHS beyond neonatal period from West Africa.

We found a prevalence of GBS in the nasopharynx $14.0 \%(162 / 1170)$, with an overall prevalence of BHS of $20.0 \%(234 / 1170)$ in NPS examined (Fig. 2a and b).

Although data on nasopharyngeal carriage of GBS during early infancy is sparse in West Africa, our findings are consistent with an earlier study in the Gambia [24], albeit in infants less than $24 \mathrm{~h}$ old, and from different body sites. 
The 13-valent pneumococcal conjugate vaccine (PCV13) has been in use in The Gambia since April 2011, before the start of this study. Few studies have reported changes in the epidemiology of bacterial carriage and diseases associated with widespread use of the pneumococcal conjugate vaccine $[25,26]$. However, the prevalence of GBS carriage and disease in the post-PCV era has not been properly described. New pneumococcal vaccines capable of inducing non-serotype dependent and broadly reacting immunity have been developed and are in early clinical trials [27]. Introduction of these new vaccines with the potential to disrupt nasopharyngeal ecology may be associated with the potential risk of species replacement including a more prominent role for related organisms such as $S$. aureus.

We previously showed that $S$. pneumoniae was positively associated with both $H$. influenzae and $M$. catarrhalis, and negatively associated with $S$. aureus in NP carriage [18]. In this study, we report for the first time, an interesting interplay of associations between GBS and other commonly studied respiratory pathogens. The negative association we found between GBS and S. pneumoniae and the positive association with $S$. aureus suggest that GBS may occupy a niche that overlaps with that of S. pneumoniae and S. aureus. It is interesting to note that the niche overlap between these pathogens may actually be quite large amongst infants from this setting as S. pneumoniae and S. aureus have both also been isolated from the oropharynx where GBS typically resides [18]. With respect to S. pneumoniae, the coexistence appears to be antagonistic, whereas the converse is true for $S$. aureus. Furthermore, no GBS isolate was co-carried with another BHS. This is an interesting finding and may suggest an exclusive pattern of cocolonization amongst BHS of different Lancefield groups, probably due to their metabolic similarities. With the development of pneumococcal protein vaccines that may wipe out carriage of $S$. pneumoniae, there's a potential risk of increase in GBS carriage with possible clinical consequences.

Although data on carriage of non-BHS in infants belonging to this age group is sparse, our findings are consistent with the existing reports from other parts of the world $[28,29]$. In a cross-sectional study such as ours, it is possible to have missed any BHS which may have a seasonal pattern of colonization, such as have been shown with S. pneumonia serotypes, 1 and 5 [30]. Confirming this would require studying a larger cohort of infants in future studies to investigate BHS carriage longitudinally.

In this study, the predominant GBS serotypes were $\mathrm{V}$ (37.6\%) similar to what was found in an earlier study in The Gambia, and II (34.6\%). In contrast to our findings, this other study conducted two decades ago did not find appreciable numbers of serotype II [24]. This suggests the existence of temporal changes in the carriage of GBS and most likely other BHS serotypes. The prevalence also varies geographically. Globally serotype III plays a major role in both early-onset and late-onset disease as well as carriage in developing and developed countries [31, 32]. However, this serotype comprised $<1 \%$ of GBS isolates in our study. There is no GBS vaccine available currently, although some vaccine candidates have been tested in preclinical studies [33]. A Novartis glycoconjugate vaccine containing serotypes Ia, Ib and III is currently undergoing phase II trial [34]. Although the carriage of GBS has not been shown to predispose to invasive disease unlike in organisms such as S. pneumoniae, it may be worthwhile for future vaccine development targeting developing countries such as The Gambia to consider the geographical differences in serotype distribution.

Latex agglutination is the conventional method for serotyping. Our data demonstrates the added utility of PCR serotyping as it resolved majority of the serotypes (19 of 21) identified as non-typeable by latex agglutination. The usefulness of such molecular serotyping techniques has been reported in the literature [35].

Penicillin is the recommended first-line of treatment for streptococcal infections, including GBS infections and all GBS isolates in this study were found to be Penicillin susceptible. Similar to other regions, we observed a high rate of tetracycline resistance [36]; however this antibiotic is not commonly used in children. Macrolides are the recommended second line treatment option. Globally, there is growing evidence of emerging resistance of GBS to erythromycin [37, 38], making it important to probe resistance patterns to this antibiotic. In our study, $<1 \%$ of GBS isolates were resistant to erythromycin; with approximately $11 \%$ showing intermediate susceptibility; an indication that macrolide antimicrobial resistance is not a major concern in our setting at this time. Our findings are similar to reports from Brazil where $4.7 \%$ of colonizing GBS isolates were resistant to erythromycin [39]; and Australia where $6.4 \%$ of GBS isolates were resistant to erythromycin [40].

Host specific factors such as age, race, breastfeeding, attendance at day care amongst others have been shown to affect colonization of $S$. pneumoniae, S. aureus, $M$. catarrhalis, and $H$. influenzae [41]. Intercurrent or past episodes of respiratory tract infections are also known to increase colonization by these pathogens [41].

Contrary to results from other studies, multiple logistic regression analyses in this study did not yield any association between GBS carriage and gender, ethnic group and overcrowding. In addition, GBS carriage was inversely associated with a history of acute respiratory infection. This finding is interesting, as it suggests that recent acute respiratory infection may have an impact 
on GBS carriage. However, we note that none of the study participants had any history of previous antibiotic use. Thus it may likely be a chance finding (although statistically significant).

\section{Conclusions}

In light of our findings, and evidence from elsewhere [25, 26], we recommend that invasive bacterial disease surveillance following introduction of new vaccines should include monitoring not just for serotype replacement but also species replacement in carriage and invasive pneumococcal disease. In addition, close surveillance monitoring is required to detect the emergence of bacterial antimicrobial resistance in the Gambia. We have shown that GBS has negative and positive associations with $S$. pneumoniae and $S$. aureus respectively. We have also shown that the GBS serotype V remains dominant in colonization of Gambian infants, and is negatively associated with the carriage of $H$. influenzae. We have also found an emergence of GBS serotype II. Although preliminary, this information is important information for future vaccine development for West African countries.

\section{Abbreviations \\ BHS, beta-haemolytic streptococci; GAS, Group A streptococcus; GBS, Group B streptococcus; GCS, Group C streptococcus; GDS, Group D streptococcus; GES, Group E streptococcus; GFS, Group F streptococcus; GGS, Group G streptococcus; Hib, Haemophilus influenzae type B; NT, non-typeable; NPS, nasopharyngeal swabs; PCV, pneumococcal conjugate vaccine}

\section{Acknowledgements}

We thank all study participants as well as clinicians, field workers and research Microbiology laboratory staff, especially technicians Amara Jah, Kaddijatou Jawneh, Maimuna Jarju and Sainabou Bojang for their assistance in processing the NPS. We are also grateful to the Leslie McGee and Centres for Disease Control and Prevention, Atlanta, USA for kindly sharing the Primers and control strains that were used in the PCR assay.

\section{Funding}

Funding was provided by the Medical Research Council Unit, The Gambia. The funders had no role in study design, data collection and analysis, decision to publish, or preparation of the manuscript.

\section{Availability of data and materials}

Data supporting the findings is contained within the manuscript.

\section{Authors' contributions}

EFN and MA conceived and designed the experiments, performed the experiments, analysed the data, wrote the paper, prepared figures and/or tables, reviewed drafts of the paper. BK, OA, FC, SJ, AB, JM, BK, MOO, IA conceived and designed the experiments, contributed reagents/materials/ analysis tools, reviewed drafts of the paper. JJ contributed reagents/ materials/analysis tools, reviewed drafts of the paper. All authors read and approved the final version of the manuscript.

\section{Competing interests}

The authors declare that they have no competing interests.

\section{Consent to publish}

Not applicable.

\section{Ethics and consent to participate}

The Gambia Government/MRC Joint Ethics Committee approved the study (reference number SCC117). Trained field workers/nurses explained the contents of the study information sheet to parents/guardians in their own language. Parents/guardians of all study participants gave written informed consent prior to enrolment.

\section{Author details}

${ }^{1}$ Vaccines and Immunity Theme, Medical Research Council Unit, Banjul, The Gambia. ${ }^{2}$ Current Address: WHO Regional Office for Africa, Brazzaville, Congo. ${ }^{3}$ Disease Control and Elimination Theme, Medical Research Council Unit, Banjul, The Gambia. ${ }^{4}$ Current Address: Infectious Diseases Epidemiology, London School of Hygiene \& Tropical Medicine, London, UK. ${ }^{5}$ Faculty of Infectious and Tropical Diseases, London School of Hygiene \& Tropical Medicine, London, UK. ${ }^{6}$ Microbiology and Infection Unit, Warwick Medical School, University of Warwick, Coventry, UK.

Received: 25 October 2015 Accepted: 19 May 2016

Published online: 27 May 2016

\section{References}

1. Ikebe T, Tominaga K, Shima T, Okuno R, Kubota H, Ogata K, et al. Increased prevalence of group A streptococcus isolates in streptococcal toxic shock syndrome cases in Japan from 2010 to 2012. Epidemiol Infect. 2015;143(04): 864-72.

2. Kuroda J, Inoue N, Satoh H, Fukuzawa R, Terakawa T, Hasegawa Y. Neonatal necrotizing fasciitis of the scrotum caused by Streptococcus agalactiae. Pediatr Int. 2015;24(10):12563.

3. Lancefield RC. A serological differentiation of human and other groups of hemolytic Streptococci. J Exp Med. 1933;57(4):571-95.

4. Zhu M, Hu Q, Mai J, Lin Z. Analysis of pathogenic bacteria and drug resistance in neonatal purulent meningitis. Zhonghua Er Ke Za Zhi. 2015; 53(1):51-6.

5. Chauhan D, Mokta K, Kanga A, Grover N, Singh D, Bhagra S. Group B streptococcal meningitis in children beyond the neonatal period in subHimalayan India. Ann Indian Acad Neurol. 2015;18(1):71-3.

6. Ku LC, Boggess KA, Cohen-Wolkowiez M. Bacterial meningitis in infants. Clin Perinatol. 2015:42(1):29-45.

7. Juncosa-Morros T, Guardia-Llobet C, Bosch-Mestres J, Dopico-Ponte E, Sanfeliu-Sala I, Sierra-Soler M, et al. Streptococcus agalactiae late-onset neonatal infections in Barcelona (1996-2010). Enferm Infecc Microbiol Clin. 2014:32(9):574-8.

8. Berkley JA, Lowe BS, Mwangi I, Williams T, Bauni E, Mwarumba S, et al. Bacteremia among children admitted to a rural hospital in Kenya. N Engl J Med. 2005;352(1):39-47.

9. Milledge J, Calis JC, Graham SM, Phiri A, Wilson LK, Soko D, et al. Aetiology of neonatal sepsis in Blantyre, Malawi: 1996-2001. Ann Trop Paediatr. 2005; 25(2):101-10.

10. Zaidi AKM, Huskins WC, Thaver D, Bhutta ZA, Abbas Z, Goldmann DA. Hospital-acquired neonatal infections in developing countries. Lancet. 2005; 365(9465):1175-88.

11. Liebana-Martos Mdel C, Cabrera-Alavargonzalez J, Rodriguez-Granger J, Miranda-Casas C, Sampedro-Martinez A, Gutierrez-Fernandez J, et al. Serotypes and antibiotic resistance patterns in beta-hemolytic Streptococcus agalactiae isolates in colonized mothers and newborns with invasive disease. Enferm Infecc Microbiol Clin. 2015;33(2):84-8.

12. Lin $\mathrm{HC}$, Chen $\mathrm{CJ}$, Chiang KH, Yen TY, Ho CM, Hwang KP, et al. Clonal dissemination of invasive and colonizing clonal complex 1 of serotype $\mathrm{VI}$ group B Streptococcus in central Taiwan. J Microbiol Immunol Infect. 2014; 11(14):00221-9.

13. Murphy TF, Bakaletz LO, Smeesters PR. Microbial interactions in the respiratory tract. Pediatr Infect Dis J. 2009;28(10 Suppl):S121-6.

14. Bogaert D, De Groot R, Hermans PW. Streptococcus pneumoniae colonisation: the key to pneumococcal disease. Lancet Infect Dis. 2004;4(3): 144-54.

15. Principi N, Marchisio P, Schito GC, Mannelli S. Risk factors for carriage of respiratory pathogens in the nasopharynx of healthy children. Ascanius Project Collaborative Group. Pediatr Infect Dis J. 1999:18(6):517-23.

16. Antonio M, Dada-Adegbola H, Biney E, Awine T, O'Callaghan J, Pfluger V, et al. Molecular epidemiology of pneumococci obtained from Gambian children aged 2-29 months with invasive pneumococcal disease during a trial of a 9-valent pneumococcal conjugate vaccine. BMC Infect Dis. 2008;8:81 
17. Hill PC, Townend J, Antonio M, Akisanya B, Ebruke C, Lahai G, et al. Transmission of Streptococcus pneumoniae in rural Gambian villages: a longitudinal study. Clin Infect Dis. 2010;50(11):1468-76.

18. Odutola A, Antonio M, Owolabi O, Bojang A, Foster-Nyarko E, Donkor S, et al. Comparison of the prevalence of common bacterial pathogens in the oropharynx and nasopharynx of Gambian infants. PLoS One. 2013;8(9): e75558.

19. Carvalho MG, Pimenta FC, Jackson D, Roundtree A, Ahmad Y, Millar EV, et al. Revisiting pneumococcal carriage by use of broth enrichment and PCR techniques for enhanced detection of carriage and serotypes. J Clin Microbiol. 2010;48(5):1611-8.

20. Imperi M, Pataracchia M, Alfarone G, Baldassarri L, Orefici G, Creti R. A multiplex PCR assay for the direct identification of the capsular type (la to IX) of Streptococcus agalactiae. J Microbiol Methods. 2010;80(2):212-4.

21. Organization $\mathbf{W H}$. Laboratory methods for the diagnosis of meningitis caused by Neisseria meningitidis, Streptococcus pneumoniae and Haemophilus influenzae. 2nd ed. 2011.

22. CLSI, editor, Clinical and Laboratory Standards Insitute. Performance Standards for Antimicrobial Susceptebility Testing: Twenty-first Informational Supplement M100-S21. Wayne, PA, USA: CLSI; 2011.

23. United Kingdom National External Quality Assessment Service [http://www. ukneqas.org.uk]

24. Suara RO, Adegbola RA, Baker CJ, Secka O, Mulholland EK, Greenwood BM Carriage of group B Streptococci in pregnant Gambian mothers and their infants. J Infect Dis. 1994;170(5):1316-9.

25. Biesbroek G, Wang X, Keijser BJ, Eijkemans RM, Trzcinski K, Rots NY, et al. Seven-valent pneumococcal conjugate vaccine and nasopharyngeal microbiota in healthy children. Emerg Infect Dis. 2014;20(2):201-10.

26. Spijkerman J, Prevaes SM, van Gils EJ, Veenhoven RH, Bruin JP, Bogaert D, et al. Long-term effects of pneumococcal conjugate vaccine on nasopharyngeal carriage of S. pneumoniae, S. aureus, H. influenzae and $M$. catarrhalis. PLoS One. 2012;7(6):25.

27. Feldman C, Anderson R. Review: current and new generation pneumococcal vaccines. J Infect. 2014;69:309-25. doi:10.1016/j.jinf.2014.06.006.

28. Collins DA, Hoskins A, Bowman J, Jones J, Stemberger NA, Richmond PC, et al. High nasopharyngeal carriage of non-vaccine serotypes in Western Australian aboriginal people following 10 years of pneumococcal conjugate vaccination. PLoS One. 2013:8(12):e82280.

29. Berkovitch M, Bulkowstein M, Zhovtis D, Greenberg R, Nitzan Y, Barzilay B, et al. Colonization rate of bacteria in the throat of healthy infants. Int J Pediatr Otorhinolaryngol. 2002;63(1):19-24.

30. Antonio M, Hakeem I, Awine T, Secka O, Sankareh K, Nsekpong D, et al. Seasonality and outbreak of a predominant Streptococcus pneumoniae serotype 1 clone from The Gambia: expansion of ST217 hypervirulent clonal complex in West Africa. BMC Microbiol. 2008;8:198.

31. Florindo C, Gomes JP, Rato MG, Bernardino L, Spellerberg B, Santos-Sanches I, et al. Molecular epidemiology of group B streptococcal meningitis in children beyond the neonatal period from Angola. J Med Microbiol. 2011; 60(Pt 9):1276-80

32. Brzychczy-Wloch M, Gosiewski T, Bulanda M. Multilocus sequence types of invasive and colonizing neonatal group B streptococci in poland. Med Princ Pract. 2014;23(4):323-30.

33. Heath PT, Feldman RG. Vaccination against group B streptococcus. Expert Rev Vaccines. 2005;4(2):207-18.

34. Novartis Streptococcus vaccine programme. https://linicaltrials.gov/ct2/ show/record/NCT02046148.

35. Brigtsen AK, Dedi L, Melby KK, Holberg-Petersen M, Radtke A, Lyng RV, et al. Comparison of PCR and serotyping of Group B Streptococcus in pregnant women: the Oslo GBS-study. J Microbiol Methods. 2015;108:31-5.

36. Beigverdi R, Jabalameli F, Mirsalehian A, Hantoushzadeh S, Boroumandi S, Taherikalani M, et al. Virulence factors, antimicrobial susceptibility and molecular characterization of Streptococcus agalactiae isolated from pregnant women. Acta Microbiol Immunol Hung. 2014;61(4):425-34.

37. Shabayek S, Abdalla S. Macrolide- and tetracycline-resistance determinants of colonizing group B streptococcus in women in Egypt. J Med Microbiol. 2014:63:1324-7.

38. Lee WT, Lai MC. High prevalence of Streptococcus agalactiae from vaginas of women in Taiwan and its mechanisms of macrolide and quinolone resistance. J Microbiol Immunol Infect. 2015;48(5):510-6.

39. Palmeiro JK, Dalla-Costa LM, Fracalanzza SE, Botelho AC, Da Silva Nogueira $\mathrm{K}$, Scheffer MC, et al. Phenotypic and genotypic characterization of group $B$ streptococcal isolates in southern Brazil. J Clin Microbiol. 2010;48(12): 4397-403.

40. Garland SM, Cottrill E, Markowski L, Pearce C, Clifford V, Ndisang D, et al. Antimicrobial resistance in group B streptococcus: the Australian experience. J Med Microbiol. 2011;60:230-5.

41. Garcia-Rodriguez JA, Fresnadillo Martinez MJ. Dynamics of nasopharyngeal colonization by potential respiratory pathogens. J Antimicrob Chemother. 2002:50:59-73.

\section{Submit your next manuscript to BioMed Central and we will help you at every step:}

- We accept pre-submission inquiries

- Our selector tool helps you to find the most relevant journal

- We provide round the clock customer support

- Convenient online submission

- Thorough peer review

- Inclusion in PubMed and all major indexing services

- Maximum visibility for your research

Submit your manuscript at www.biomedcentral.com/submit
Biomed Central 